\title{
Robust Stereoscopic Head Pose Estimation in Human-Computer Interaction and a Unified Evaluation Framework
}

\author{
Georg Layher ${ }^{1}$, Hendrik Liebau ${ }^{1}$, Robert Niese ${ }^{2}$, Ayoub Al-Hamadi ${ }^{2}$, \\ Bernd Michaelis $^{2}$, and Heiko Neumann ${ }^{1}$ \\ 1 University of Ulm, Institute of Neural Information Processing \\ \{georg. layher, hendrik. liebau, heiko.neumann\}@uni-ulm.de \\ 2 Otto-von-Guericke University Magdeburg, IESK \\ \{robert.niese, ayoub.al-hamadi, bernd.michaelis\}@ovgu.de
}

\begin{abstract}
The automatic processing and estimation of view direction and head pose in interactive scenarios is an actively investigated research topic in the development of advanced human-computer or human-robot interfaces. Still, current state of the art approaches often make rigid assumptions concerning the scene illumination and viewing distance in order to achieve stable results. In addition, there is a lack of rigorous evaluation criteria to compare different computational vision approaches and to judge their flexibility. In this work, we make a step towards the employment of robust computational vision mechanisms to estimate the actor's head pose and thus the direction of his focus of attention. We propose a domain specific mechanism based on learning to estimate stereo correspondences of image pairs. Furthermore, in order to facilitate the evaluation of computational vision results, we present a data generation framework capable of image synthesis under controlled pose conditions using an arbitrary camera setup with a free number of cameras. We show some computational results of our proposed mechanism as well as an evaluation based on the available reference data.
\end{abstract}

Keywords: head pose estimation, stereo image processing, human computer interaction.

\section{Introduction}

Human-centered computing has paved the ground for the integration of new technologies into the development of natural and intuitive-to-use interfaces for advanced human-computer interaction (HCI). The visual modality is an important channel for non-verbal communication transmitting social signals which contain rich behavioral cues concerning attention, communicative initiative, empathy, etc. (Pentland 2007; Vinciarelli et al. 2008). Such cues are conveyed through various behavioral signals and combinations thereof which allow their automatic analysis by computers (Corso et al. 2008; Jacob 1996; Turk 2004). These social signals can be interpreted to reason about the user state or intention in a contextdependent fashion to launch differentiated communicative or social reactions in 
next generation HCI systems. Here we focus on one of the most meaningful cues concerning the user's focus of attention relative to the observer's (camera's) view direction which can be derived from an actor's head pose direction. Vision-based estimation of the human head pose received more and more attention over the last decade and a wealth of methods and mechanisms have been proposed. An overview is presented in (Murphy-Chutorian \& Trivedi 2009). Still, the robustness of many of these approaches suffer from large errors and redundancies in the feature extraction and matching mechanisms, variations in illumination conditions, or size changes due to variable camera-actor distances. Here, we propose a new stereo matching approach that operates on features derived from hierarchical processing of images pairs in a biologically inspired architecture of static form processing. Stereo matching is subsequently driven by learned intermediate-level image features which allow robust matching and fast false targets reduction.

Considering the evaluation of methods, a large number of different approaches entail numerous different training and test data sets. For example, MurphyChutorian \& Trivedi (2009) quote no less than 14 different databases. However, such data sets are often somehow adapted to support the particular research focus of a given approach which leads to restrictions in the variation of free parameters. This makes it virtually impossible to compare fundamentally different computational methods for estimating head poses using only one of the currently available datasets. In addition to our newly developed method for pose estimation, we propose a data generation framework that enables us to generate input for any kind of head pose estimation approach and thus allowing a comprehensive comparison.

The rest of the paper is structured as follows. In Section 2 we introduce a new stereo matching mechanism for robust head pose estimation. Section 3 details the suggested approach for the development of an evaluation framework. In Section 4 we demonstrate the functional significance of the proposed computational method in conjunction with the evaluation framework.

\section{Stereo Head Pose Estimation}

We propose a robust approach to estimate the 3-dimensional (3D) head pose from stereoscopic image pairs. The approach mainly consists of three processing stages. First, the images of a stereo pair are initially processed to detect and localize facial features of different complexity and at different image scales. The processing scheme employed here follows a biologically inspired model architecture of the cortical ventral pathway in the primate visual system (Riesenhuber \& Poggio 1999). Second, the different features detected in the left and right image frames of a stereo pair are subsequently matched. Our approach is inspired by recent findings that visual features with intermediate size and complexity are superior in terms of their specificity and their misclassification probability (Ullman et al. 2002). Following this observation we propose making use of intermediate level features as learned by the previously mentioned processing hierarchy. Using such complex features reduces the numbers of false targets in the stereo correspondence problem and thus quickly resolves the matching ambiguities. At the 
same time the features used still allow spatial resolutions of sufficient detail. Correspondence is established by using a simple correlation-based matching approach. Third, the facial feature localization and the subsequent calculation of disparity values between each pair of corresponding features allow to reconstruct the $3 \mathrm{D}$ location of a patch of matched image regions. The estimated $3 \mathrm{D}$ surface information in world coordinates is then used to fit a so-called facial plane (defined by the eyes and the mouth) to estimate the pose angle. In the following, we briefly describe the object recognition framework used for the localization of the face and the enclosed features. Subsequently, we address the issue of how the positions of the features can be used for an efficient and robust estimation of the head pose.

\subsection{Hierarchical Feature Extraction}

For the detection of the head and the facial features, we implemented a slightly modified version of the biologically inspired object-recognition model proposed by Mutch \& Lowe (2008). It is based on a model by Serre et al. (2005), which in turn extends the above-mentioned "HMAX" model proposed by Riesenhuber \& Poggio (1999). The proposed model architecture utilizes alternating layers of so-called simple $(\mathrm{S})$ and complex $(\mathrm{C})$ cells. In a nutshell, simple cells perform a local linear filtering operation, i.e. a convolution, and combine spatially adjacent elements into a high-order feature. Complex cells, on the other hand, increase position and scale invariance by a nonlinear pooling operation. As sketched in Fig. 1] our model variant consists of five different layers of processing. The input layer transforms the original image $\left(I_{i n}\right)$ into a pyramidal representation of different spatial scales $\left(I_{\text {scale }}\right)$ through proper low-pass filtering and downsampling,

$$
I_{\text {scale }}(x, y)=\left(I_{i n}(x, y) \star \frac{1}{2 \pi \sigma^{2}} \exp \left(-\frac{x^{2}+y^{2}}{2 \sigma^{2}}\right)\right) \cdot I I I_{\Delta x, \Delta y}(x, y)
$$

where $\star$ denotes the spatial convolution operator, $\sigma$ denotes the width of the Gaussian low-pass filter (for reducing the signal frequency content), and $I I I(\cdot)$

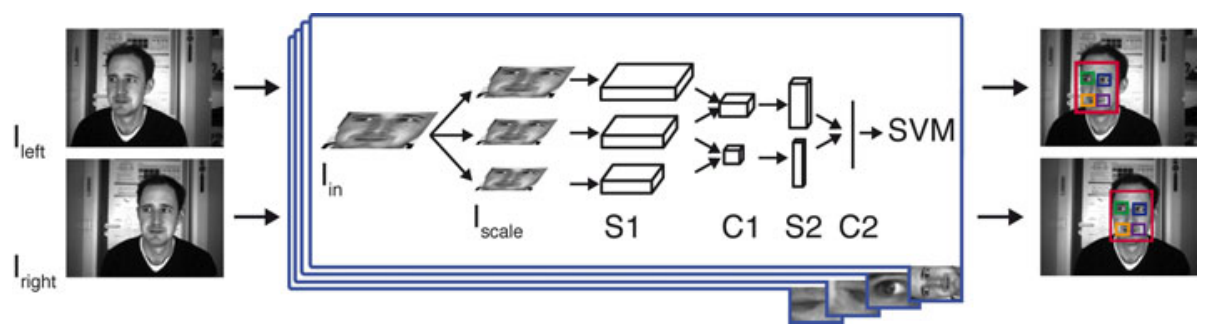

Fig. 1. In the first processing step, a face and its correspondent are detected in a stereo image pair. The same object-recognition framework is then used to localize both eyes and the mouth corners within the two facial regions. Over all, the framework has to be trained on four different kinds of features (faces, eyes, left and right mouth corners). 
is the down-sampling function (Shah function, Bracewell 1978) with $\Delta x, \Delta y$ denoting the sampling width on a rectangular grid. Each scale representation is then convolved with 2D Gabor filters of four orientations. These filters resemble the shape of receptive field profiles of cells in the mammalian visual cortex (Hubel \& Wiesel 1959). The Gabor filter kernels are generated by Gaussian window functions modulated by oriented sinusoidal wavefronts

$$
g_{\sigma, \omega}(x, y)=\exp \left(-\frac{x^{2}+y^{2}}{2 \sigma^{2}}\right) \cdot f_{\text {wave }}\left(\omega_{\mathbf{0}}^{T} \cdot(x, y)\right)
$$

with $f_{\text {wave }}(x)=\cos (x)$ or $f_{\text {wave }}(x)=\sin (x)$ (even and odd modulation functions), $\sigma$ again denotes the width of the Gaussian, and $\omega_{0}=\left(\omega_{x 0}, \omega_{y 0}\right)$ denotes the modulating wave of a given frequency and orientation. Convolution of these kernels from the bank of filters with orientations and scales,

$$
I_{\sigma, \omega}(x, y)=I_{\text {scale }}(x, y) \star g_{\sigma, \omega}(x, y)
$$

generates output activations in layer S1 leading to a 4D feature representation (position, scale and orientation). Unlike Serre et al. (2005) receptive fields of different sizes are modeled here through the image pyramid instead of Gabor filters tuned to different spatial frequencies. Consequently, the number of necessary convolution operations is reduced. Layer C1 cells pool the activities of S1 cells in the same orientation channel over a small local neighborhood. This leads to a locally increasing scale, position, and size invariance, while decreasing the spatial extent of the representation. The intermediate feature layer S2 performs a simple template matching of patches of $\mathrm{C} 1$ units and a number of learned prototypes utilizing a sliding window approach (following the suggestion by Mutch \& Lowe 2008). In a nutshell, the learning algorithm selects the most descriptive and discriminative prototypes among an exhaustive number of $\mathrm{C} 1$ patches that were randomly sampled during the learning process. Disregarding the learning process the prototypes resemble spatial filters of higher feature complexity concerning their response selectivity. The correlation responses of the prototypes with the input patterns generate a feature vector of corresponding response amplitudes. In the last layer the S2 prototype responses are pooled over all positions and scales by choosing the maximum value for each prototype to yield a single feature vector as $\mathrm{C} 2$ response. These vectors finally serve as input to a linear support vector machine (SVM) that is used for the classification of faces, as well as the associated facial features.

\subsection{Stereo Matching}

Input images of a stereo pair are processed individually by using the hierarchical processing architecture sketched above. Using pairs of stereo images with slightly different views on the scene the next step is to automatically determine the matching points from one image with the corresponding points in the second image of the pair. The feature sets available from the preprocessing stage range from small localized features at the finest resolution to intermediate size patches 

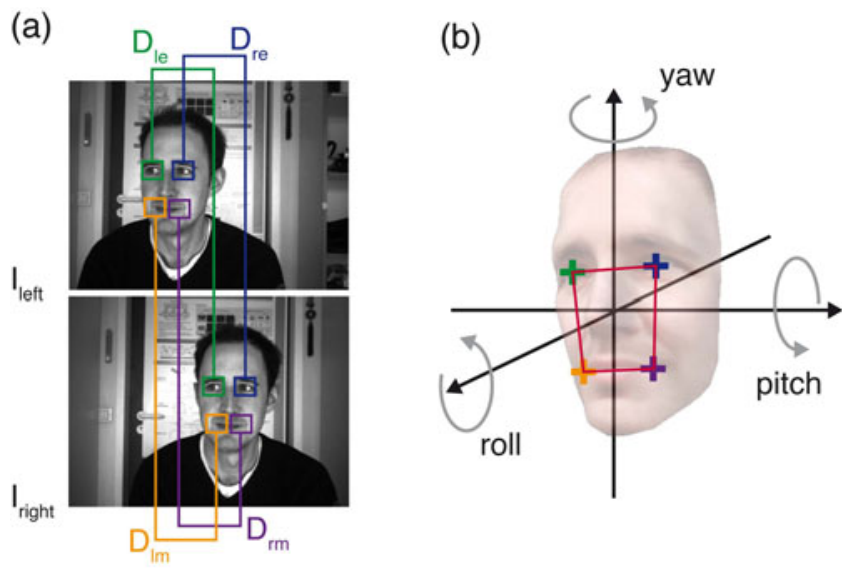

Fig. 2. Disparity values are calculated for all four facial features (a). The infered information about the 3D-positions of the features is used to determine the orientation of the facial plane (b).

up to the full image at the coarsest resolution level of the pyramid. Most traditional stereo matching approaches operate on fine resolutions utilizing certain similarity measures for initial correspondence findings. Pyramid matching approaches using a coarse-to-fine scheme make use of differently filtered versions of the input (compare, e.g., Szeliski 2011). Unlike these approaches, we make use of the findings of Ullman et al. (2002) that intermediate level image fragments are most distinctive in providing structural information about the presence of features. More precisely, a face fragment of intermediate size provides reliable indicators of face presence and locality. For the increase in reliability one has to pay the price that the likelihood of appearance of the same pattern in a new face image is low. A small fragment, corresponding to a local filter response, increases the likelihood but at the same time reduces the specificity (increase of uncertainty). This property is also reflected in the auto-correlation surface $E_{A C}$ using a sum-of-squared-distance measure: For a small fragment of low complexity the correlation surface is noisy and rather unspecific while for intermediate complexity it becomes more pronounced and less error-prone due to the noise. The intermediate-size fragments still allow localization in the image such that, e.g., the left and right eye can be distinguished (results not shown). Considering the correspondence finding in stereo images these intermediate-level fragments help to improve the stability of finding candidate matches and reduce the false-targets uncertainty.

Our matching approach makes use of a simple correlation-based similarity measure using image fragments of intermediate complexity. The correlation measure is maximized starting with the reference template fragment and using a search window along epipolar lines in the left and right image (Barnard \& Fischler 1982, Hannah 1988). It is important to stress, that we do not need to 
estimate a dense disparity map. Instead, we need to take into account only few facial features (namely the eyes and the mouth corners) and their stereo correspondence to subsequently estimate the spatial head orientation (see Fig. 22). This considerably reduces the computational costs of the correspondence finding and pose estimation process.

\subsection{Estimating 3D Head Pose Orientation}

Given the disparity values of the left and right eye and the corners of the mouth, as well as the focal length and the baseline of the stereo camera system, we are able to estimate a plane that fits the four spatial points of eye and mouth in 3D. The plane is fitted using a least-squares approach minimizing the distance of the projections of the four $3 \mathrm{D}$ coordinates. The orientation of the resulting plane is then used as an estimation of the head pose (see Fig. $2 \mathrm{~b}$ ).

\section{Data Generation Framework}

For the generation of image sequences as training and testing data we use a textured generic 3-D model. Based on three key points, i.e. left and right eye plus upper lip point, the generic model is adapted to a specific face. The key points are gathered from a frontal face image of a given subject using the Viola \& Jones (2004) Haar-like feature detection algorithm with cascades provided by Santana (2005). Based on the actual inter-ocular distance (which must be provided) and the ratio of face width and height estimated from the detected image points, i.e. eye distance and distance between eye axis and mouth, the generic 3-D model is scaled. The texture is assigned to the frontal image of the face. The availability of textured 3-D models enables quick OpenGL rendering of images under controlled pose conditions (see Fig 3) using an arbitrary camera setup with a free number of cameras. This way image material can be generated for accuracy testing of pose estimation methods. Also training data can be created for holistic pose estimation approaches. An example for a two camera setup is given in Figure 3. For the parameterization of the virtual cameras we chose to emulate a real camera setup in our vision lab. This has two advantages; on the one hand we determined the camera viewing parameters through calibration of the real cameras. On the other hand, after simulation and accuracy testing, we can directly turn our method into real application, using the same camera setup.

\section{Estimation Results}

The proposed stereo head pose estimation approach was tested under two different conditions. At first we used a local head pose database in combination with the framework described in Section 3 to generate test data with known ground truth. In Fig. 3] an exemplary picture of the database, as well as the resulting stereo image pairs for different head poses are shown. The estimated 

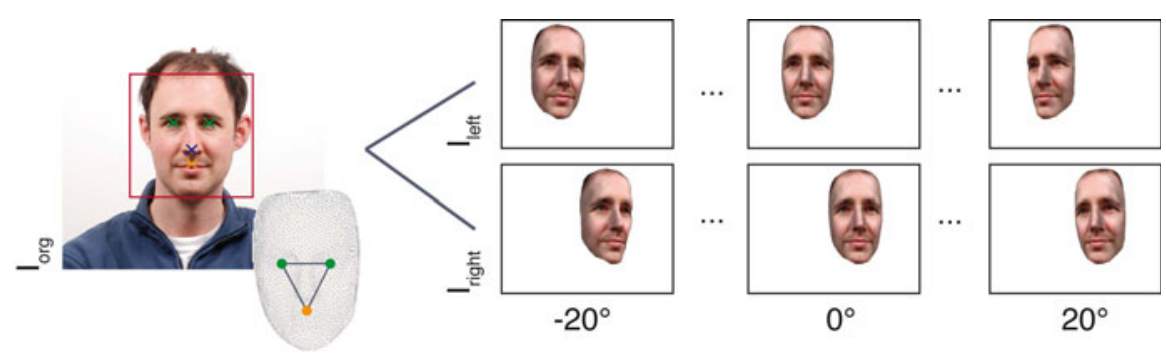

Fig. 3. A local database in which head poses were systematically w.r.t. yaw and pan angle served as input for the generation of artificial images of different head poses (as described in Section 3 the database is available via http://www.uni-ulm.de/in/neuroinformatik/mitarbeiter/g-layher; Weidenbacher et al. 2007). The virtual camera setup was adjusted to the specification of the Bumblebee ${ }^{\circledR} 2$ Stereo Vision Camera System (Point Grey Research Inc.) which was used in a real world testing scenario (Fig. 4b). The artificially generated stereo images were used to evaluate the head pose estimation approach as described in Section 2.

head pose is displayed in Fig. 4a. For the test data shown in Fig. 3, the estimation error increases for larger yaw angles, but never exceeds $3^{\circ}$ over head poses in the range of $\left[-20^{\circ}, 20^{\circ}\right]$. Secondly, we used a sequence of real world images to test the accuracy of the estimated head pose under unrestricted conditions. The actual head pose within the sequence was unknown, but the subject was told to systematically rotate his head from left to right. As shown in Fig. 4b, the estimated head pose reflects that fact. Note that the underlying classifiers used for the localization of the head and the facial features were all trained using the FERET Database (Phillips et al. 2000) and thus have no association to the test data at all. Fig. $4 c$ shows the capability of the proposed approach under varying camera-actor distances.

\section{Discussion and Conclusion}

In this study we have demonstrated that online stereo head pose estimation can be achieved by utilizing features of intermediate complexity. Stereoscopic head pose estimation is simplified by matching only significant facial features, thus keeping the disparity map sparse. The use of intermediately complex templates for matching left and right image pairs allows to use a simple correlation-based criterion as similarity measure. The distinctiveness of the features used greatly reduces the matching ambiguities to improve the reliability of the correspondence estimation. While many previous approaches assume that the observer keeps his viewing distance in a restricted range, the approach here allows large changes in viewing distance (or scale) while at the same time continuously varying the view direction (yaw and pitch) and changing the facial mimics. The approach meets several design criteria that were suggested by Murphy-Chutorian \& Trivedi 
(a)

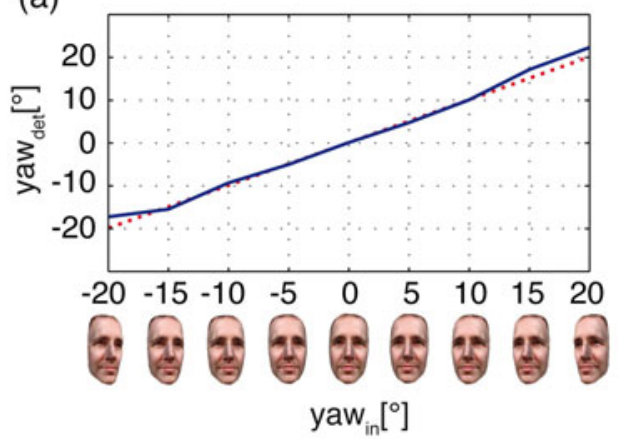

(b)

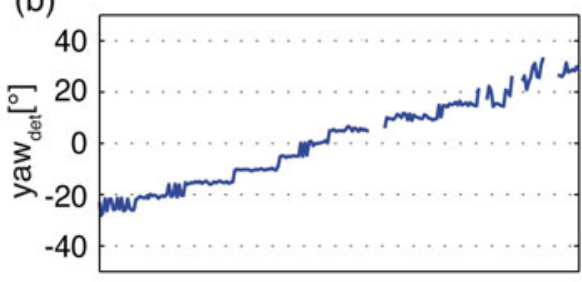

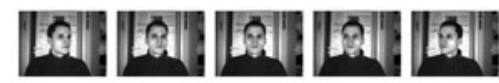

(c)
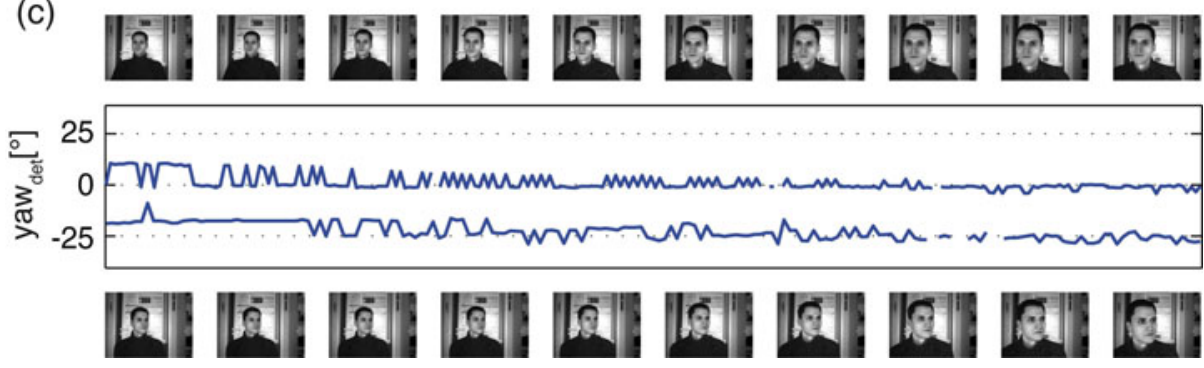

Fig. 4. Head pose estimation results. (a) An artificially generated sequence of stereo images (using the 3D face model desribed in Section [3) contains pictures of horizontal head poses in a range of $-20^{\circ}$ to $+20^{\circ}$, in steps of $5^{\circ}$ (yaw angle) used as input (ground truth). The resulting head pose estimates lead to an error that is less than $3^{\circ}$ (with large yaw angles producing larger errors). (b) A sequence of real world stereo images was obtained using the Bumblebee ${ }^{\circledR} 2$ Camera System. The subject was instructed to rotate his head continuously from left to right, thus yielding different yaw angles for head poses. Even though no ground truth data is available, it can be seen that the estimated head pose follows the pose characteristic of the head. (c) The capability of the proposed approach under varying camera-actor distances was tested using two real world sequences with different but constant yaw angles (as shown above and below the plot). As can be seen, there is only a little change in the estimated head poses for differing distances. Note that the apparently large differences of the estimated head poses in the left half of the plot are caused by a mere difference of one pixel in the disparity values. This reflects the fact, that with an increasing camera-actor distance, the pixel resolution is more and more restricting the number of distinguishable head poses.

(2009), namely accuracy (achieving pose estimates with less than $3^{\circ}$ mean absolute error for yaw $/$ pitch angles below $+/-40^{\circ}$ ), autonomy of processing without manual initialization or feature localization, invariance against person identity and illumination, and independence of the spatial resolution and viewer distance. Apart from the technical approach of pose estimation, we also suggested a strategy to evaluate the method by using a textured 3D model to flexibly generate synthetic ground truth data for arbitrary camera views. 
In all, we suggest that the approach makes a valuable contribution to build more flexible vision-based conversational systems in human-computer interaction and affective communication. For example, the approach presented here allows continuous and reliable head pose estimation and the recognition of head gestures analyzing social signals in communication (Morency \& Darrell 2004: Vinciarelli et al. 2008, Pentland 2007). Other approaches also used stereo-based head pose estimation. For example, Voit et al. (2006) estimate head poses in meeting room scenarios using multiple cameras, while Morency et al. (2006) estimate pose information to detect conversational turns for an embodied conversational agent. In these scenarios the variation of viewer distance and thus image resolution can be assumed to change only within a limited range and that the luminance conditions can be controlled. Furthermore the precision of the focus of attention (as estimated from the head pose direction) was not evaluated in detail. Here, we suggest an approach that successfully handles these variable conditions and allows further applications in interactive scenarios. For example, we are interested to already set an interactive companion system into an alert condition when a person approaches. During the course of approaching the person could be analyzed in terms of communicative social signals and the vision-based system might properly react ahead of the first interaction. We will address this in future investigations.

Acknowledgments. This research has been supported by a Grant from the Transregional Collaborative Research Center SFB/TRR62 "Companion-Technology for Cognitive Technical Systems" funded by the German Research Foundation (DFG). Portions of the research in this paper use the FERET database of facial images collected under the FERET program, sponsored by the DOD Counterdrug Technology Development Program Office.

\section{References}

Pentland, A.: Social Signal Processing. IEEE Signal Processing Magazine 24(4), 108$111(2007)$

Vinciarelli, A., Pantic, M., Bourlard, H., Pentland, A.: Social Signals, their Function, and Automatic Analysis: a Survey. In: Proceedings of the 10th international conference on Multimodal interfaces (ICMI 2008), pp. 61-68. ACM, New York (2008)

Corso, J.J., Ye, G., Burscbka, D., Hager, G.D.: A Practical Paradigm and Platform for Video-Based Human-Computer Interaction. IEEE Computer 41(5), 48-55 (2008)

Jacob, R.: Human-Computer Interaction: Input Devices. ACM Computing Surveys 28(1), 177-179 (1996)

Turk, M.: Computer Vision in the Interface. Commun. ACM. 47(1), 60-67 (2004)

Murphy-Chutorian, E., Trivedi, M.M.: Head Pose Estimation in Computer Vision: A Survey. IEEE Trans. on Pattern Analysis and Machine Intelligence 31(4), 607-626 (2009)

Riesenhuber, M., Poggio, T.: Hierarchical Models of Object Recognition in Cortex. Nature Neuroscience 2(11), 1019-1025 (1999)

Ullman, S., Vidal-Naquet, M., Sali, E.: Visual Features of Intermediate Complexity and their Use in Classification. Nature Neuroscience 5(7), 682-687 (2002) 
Mutch, J., Lowe, D.G.: Object Class Recognition and Localization Using Sparse Features with Limited Receptive Fields. Int. J. Comput. Vision 80(1), 45-57 (2008)

Serre, T., Wolf, L., Poggio, T.: Object Recognition with Features Inspired by Visual Cortex. In: Proceedings of the 2005 IEEE Computer Society Conference on Computer Vision and Pattern Recognition (CVPR 2005), pp. 994-1000. IEEE Computer Society, Washington (2005)

Bracewell, R.N.: The Fourier Transform and its Applications. McGraw-Hill, Columbus (1978)

Hubel, D.H., Wiesel, T.N.: Receptive Fields of Single Neurones in the Cat's Striate Cortex. Journal of Physiology 148(3), 574-591 (1959)

Szeliski, R.: Computer Vision - Algorithms and Applications. Springer, London (2011)

Barnard, S.T., Fischler, M.A.: Computational Stereo. ACM Computing Surveys (CSUR) 14(4), 553-572 (1982)

Hannah, M.J.: Digital Stereo Image Matching Technique. In: Proc. XVIth ISPRS Congress (Int'l Soc. for Photogrammtery and Remote Sensing), Commission III, Kyoto, Japan, vol. XXVII, Part B3, pp. 280-293 (1988)

Castrillón-Santana, M., Lorenzo-Navarro, J., Déniz-Suárez, O., Isern-González, J., Falcón-Martel, A.: Multiple Face Detection at Different Resolutions for Perceptual User Interfaces. In: Marques, J.S., Pérez de la Blanca, N., Pina, P. (eds.) IbPRIA 2005. LNCS, vol. 3522, pp. 445-452. Springer, Heidelberg (2005)

Viola, P., Jones, M.J.: Robust Real-Time Face Detection. Int. J. Comput. Vision 57(2), 137-154 (2004)

Phillips, P.J., Moon, H., Rizvi, S.A., Rauss, P.J.: The FERET Evaluation Methodology for Face-Recognition Algorithms. IEEE Trans. on Pattern Analysis and Machine Intelligence 22(1), 1090-1104 (2000)

Morency, L.-P., Darrell, T.: From Conversational Tooltips to Grounded Discourse: Head Pose Tracking in Interactive Dialog Systems. In: Proceedings of the 6th International Conference on Multimodal Interfaces, pp. 32-37. ACM, New York (2004)

Lades, M., Vorbrüggen, J.C., Buhmann, J., Lange, J., von der Malsburg, C., Wurtz, R.P., Konen, W.: Distortion Invariant Object Recognition in the Dynamic Link Architecture. IEEE Trans. on Computers 42(3), 300-311 (1993)

Voit, M., Nickel, K., Stiefelhagen, R.: A Bayesian Approach for Multi-View Head Pose Estimation. In: Proceedings of the 2006 IEEE International Conference Multisensor Fusion and Integration for Intelligent Systems (MFI 2006), pp. 31-34. IEEE Computer Society, Washington (2006)

Morency, L.-P., Christoudias, C.M., Darrell, T.: Recognizing Gaze Aversion Gestures in Embodied Conversational Discourse. In: Proceedings of the 8th International Conference on Multimodal Interfaces, ICMI 2006 (2006)

Weidenbacher, U., Layher, G., Strauss, P.-M., Neumann, H.: A Comprehensive Head Pose and Gaze Database. In: 3rd IET International Conference on Intelligent Environments, IE 2007 (2007) 